\title{
Dayside temperatures in the Venus upper atmosphere from Venus Express/VIRTIS nadir measurements at $4.3 \mu \mathrm{m}^{\star}$
}

\author{
J. Peralta ${ }^{1,2}$, M. A. López-Valverde ${ }^{2}$, G. Gilli ${ }^{3}$, and A. Piccialli ${ }^{4,5}$ \\ ${ }^{1}$ Institute of Space and Astronautical Science-Japan Aerospace Exploration Agency 3-1-1, Yoshinodai, 252-5210 Chuo-ku, \\ Sagamihara Kanagawa, Japan \\ e-mail: javier.peralta@jaxa.jp \\ 2 Instituto de Astrofísica de Andalucía (IAA-CSIC), Glorieta de la Astronomía s/n, 18008 Granada, Spain \\ ${ }^{3}$ Laboratoire de Météorologie Dynamique, CNRS, 75006 Paris, France \\ ${ }^{4}$ LATMOS - UVSQ/CNRS/IPSL, 11 Bd d'Alembert, 78280 Guyancourt, France \\ 5 LESIA, Observatoire de Paris/CNRS/UPMC/Univ. Paris Diderot, 92195 Meudon, France
}

Received 14 August 2015 / Accepted 17 October 2015

\begin{abstract}
In this work, we analysed nadir observations of atmospheric infrared emissions carried out by VIRTIS, a high-resolution spectrometer on board the European spacecraft Venus Express. We focused on the ro-vibrational band of $\mathrm{CO}_{2}$ at $4.3 \mu \mathrm{m}$ on the dayside, whose fluorescence originates in the Venus upper mesosphere and above. This is the first time that a systematic sounding of these non-local thermodynamic equilibrium (NLTE) emissions has been carried out in Venus using this geometry. As many as 143,218 spectra have been analysed on the dayside during the period 14/05/2006 to 14/09/2009. We designed an inversion method to obtain the atmospheric temperature from these non-thermal observations, including a NLTE line-by-line forward model and a pre-computed set of spectra for a set of thermal structures and illumination conditions. Our measurements sound a broad region of the upper mesosphere and lower thermosphere of Venus ranging from $10^{-2}-10^{-5} \mathrm{mb}$ (which in the Venus International Reference Atmosphere, VIRA, is approximately $100-150 \mathrm{~km}$ during the daytime) and show a maximum around $195 \pm 10 \mathrm{~K}$ in the subsolar region, decreasing with latitude and local time towards the terminator. This is in qualitative agreement with predictions by a Venus Thermospheric General Circulation Model (VTGCM) after a proper averaging of altitudes for meaningful comparisons, although our temperatures are colder than the model by about $25 \mathrm{~K}$ throughout. We estimate a thermal gradient of about $35 \mathrm{~K}$ between the subsolar and antisolar points when comparing our data with nightside temperatures measured at similar altitudes by SPICAV, another instrument on Venus Express (VEx). Our data show a stable temperature structure through five years of measurements, but we also found episodes of strong heating/cooling to occur in the subsolar region of less than two days.
\end{abstract}

Key words. molecular processes - radiation mechanisms: non-thermal - radiative transfer - instrumentation: spectrographs methods: data analysis - planets and satellites: atmospheres

\section{Introduction}

The upper mesosphere and lower thermosphere of Venus (or jointly, UMLT) are defined in this work as the altitude ranges $90-120 \mathrm{~km}$ and $120-150 \mathrm{~km}$ above the surface, respectively. Concretely, the zone $90-120 \mathrm{~km}$ is of great interest for being a transition region in terms of atmospheric dynamics, radiation, and photochemistry (Bougher et al. 2002; Gilli et al. 2015). The transition from the retrograde superrotating zonal (RSZ) flow to the subsolar-to-antisolar (SS-AS) circulation occurs in this zone (Bougher et al. 2006); the $\mathrm{CO}_{2}$ heating and cooling in the IR dominate the radiative balance up to about 120-130 km (Roldán et al. 2000), and the absorption/scattering processes of the Venus high-altitude haze also play an important role at these altitudes (Wilquet et al. 2009). Since the high thermal contrasts reported between day- and nightside must be responsible for the pressure gradients driving the SS-AS circulation (Bougher et al. 2006),

\footnotetext{
* The table with numerical data and averaged temperatures displayed in Fig. 7A provided as a CSV data file is only available at the CDS via anonymous ftp to

cdsarc.u-strasbg.fr (130.79.128.5) or via

http://cdsarc.u-strasbg.fr/viz-bin/qcat?J/A+A/585/A53
}

knowledge of the horizontal distribution of neutral gas temperature is essential to understand the general circulation of the Venus atmosphere, to improve numerical models, and to perform aeronomy calculations. Unfortunately, the thermal structure is poorly known at these altitudes, mainly because of its difficult accessibility. In situ measurements by spacial probes are too limited in both spatial and temporal coverage (Keating et al. 1985), while ground-based and remote sensing observations are scarce to date and are only allowed to sense a restricted set of altitudes (Bougher et al. 2006). New measurements made by various instruments on the VEx spacecraft have provided important advances concerning the vertical characterization of the Venus atmospheric thermal structure, but this applies mostly below about $100 \mathrm{~km}$. For example, radio occultation probed from 40 to $90 \mathrm{~km}$ (Tellmann et al. 2009, 2012), while sensing the night-time emission at selected IR wavelengths, allows us to sense between 65 and $96 \mathrm{~km}$ (Grassi et al. 2010; Migliorini et al. 2012; GarateLopez et al. 2015). Temperatures in the altitude range between 90 and $150 \mathrm{~km}$ have been inferred, but only on the nightside with stellar occultation (Piccialli et al. 2015), and in the morning and evening terminators with solar occultation techniques (Mahieux et al. 2015b). Although sparse in the horizontal (lat, local time), 
dayside temperatures between 100 and $150 \mathrm{~km}$ have been obtained from limb observations of the infrared non-local thermodynamic equilibrium (NLTE) emissions of CO at $4.7 \mu \mathrm{m}$ (Gilli et al. 2015). A few other observations available in the UMLT are dispersed (Sonnabend et al. 2012; Krasnopolsky 2014) or focused at selected local times (Mahieux et al. 2015a). Hence, the temperatures in the upper mesosphere and lower mesosphere and, in particular, their horizontal distribution have not been properly issued to date.

$\mathrm{CO}_{2}$ is the most abundant molecule in the atmosphere of Venus and its infrared emissions are known to be very strong during the daytime because of solar fluorescence, which is particularly the case in NLTE situations. These are important in the upper atmosphere, where pressure and therefore the frequency of molecular collisions are so low that radiation dominates the states' populations (Dickinson 1972; López-Puertas \& Taylor 2001). NLTE also affects both cooling and heating processes, and consequently the thermal state and pressure gradients that drive atmospheric motions in the upper atmosphere. Where important, NLTE effects need to be considered in the correct retrieval of atmospheric temperature and species abundances. The Visible and InfraRed Thermal Imaging Spectrometer (VIRTIS) instrument (Drossart et al. 2007) on board VEx (Svedhem et al. 2007) is capable of obtaining moderate resolution spectra of the Venus atmosphere using one of its channels (VIRTIS-H). In this work, we analysed nadir observations carried out by this channel, focusing on the ro-vibrational band of $\mathrm{CO}_{2}$ at $4.30 \mu \mathrm{m}$ in the dayside, whose fluorescence peaks within 100-140 km in height (López-Valverde et al. 2007). We carry out systematic analysis and retrieval of these NLTE emissions in this work, similar to a recent study using NLTE limb observations (Gilli et al. 2009, 2015): first, the VIRTIS-H spectra are examined and compared with the results from our NLTE model for Venus, and, second, a NLTE retrieval scheme is designed and applied to these data to infer the global horizontal distribution of daytime temperature in the UMLT of Venus with unprecedented detail.

\section{Measurements and NLTE modelling}

The instrument VIRTIS on board VEx (Drossart et al. 2007; Gilli et al. 2015) is a dual instrument consisting of two channels named VIRTIS-H and VIRTIS-M. The first one is an infrared echelle spectrometer with a spectral ranging $1.8-5.0 \mu \mathrm{m}$ and a moderate spectral resolution $(R \sim 1200)$, and the second is a mapping spectrometer working in the visible $(0.27-1.1 \mu \mathrm{m})$ and in the infrared (1.0-5.2 $\mu \mathrm{m})$ with lower spectral resolution $(R \sim 200)$, but a much wider field of view. Despite the clear advantages of using the spectral cubes from VIRTIS-M to infer the horizontal distribution of temperatures, we discarded VIRTIS$\mathrm{M}$ data due to problems of calibration in the spectral range of interest. We used the complete database of nadir spectra taken by VIRTIS-H during the whole VEx mission of about five years of observations, implying a total of 200, 036 spectra after restricting the solar zenith angle (SZA) and emission angle (EA) to values lower than $80^{\circ}$ (due to noise). This data set is much larger than the VIRTIS-H set of limb observations. As a result of VEx eccentric orbits, the projected spatial resolution of VIRTIS$\mathrm{H}$ observations can change dramatically within the same orbit, varying from a few hundreds of meters to dozens of kilometers, as the satellite moves from periapsis to the apoapsis, respectively (Gilli et al. 2015). Hence, caution must be taken for limb observations. However, in nadir viewing this is not that critical; we show later that the dimension of the spatial averages used in this work are larger than the field of view of the observations with
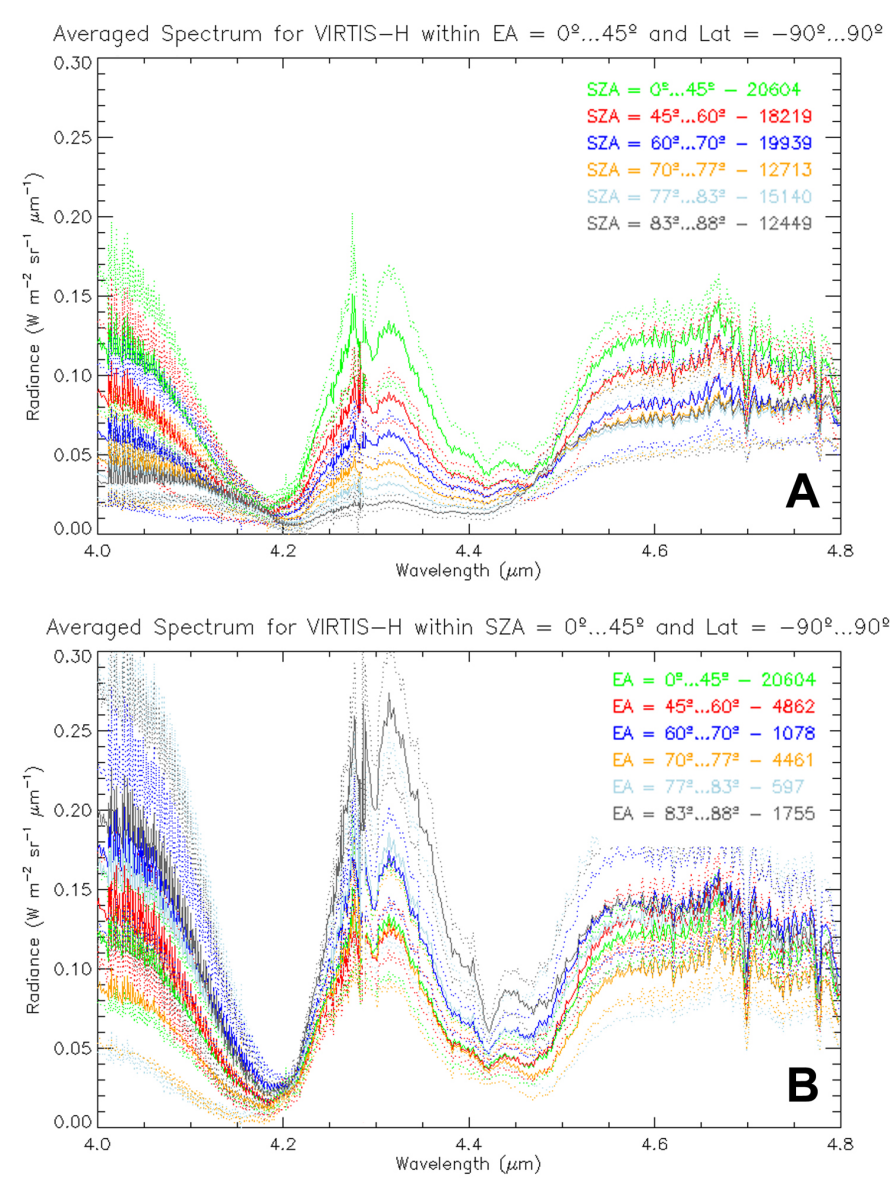

Fig. 1. Averaged spectra taken by VIRTIS-H and for all latitudes during Medium Term Planning covering several orbits of VEx (MTP001). Sets of spectra are shown for different intervals of SZA fixing the EA (A), and different intervals of EA fixing the SZA $(\mathbf{B})$.

worse spatial resolution. The VIRTIS-H spectra are subdivided in eight spectral orders, with 432 elements in each one, covering infrared wavelengths from 1.88 to $5.03 \mu \mathrm{m}$ (Drossart et al. 2007). We only used the order covering 4.01-5.03 $\mu \mathrm{m}$ (2000$2500 \mathrm{~cm}^{-1}$ ) in this work, which includes the strong NLTE $\mathrm{CO}_{2}$ emission at $4.3 \mu \mathrm{m}$. The spectral resolution in our order is about $2 \mathrm{~cm}^{-1}$ and the sampling step is $1 \mathrm{~cm}^{-1}$ which, in contrast to the $\mathrm{CO}$ band at $4.7 \mu \mathrm{m}$, inhibits the separation of the $\mathrm{CO}_{2}$ rotational lines and vibrational bands (Gilli et al. 2015). In contrast to the limb data used by Gilli et al. (2015), we cannot retrieve vertical information from this $\mathrm{CO}_{2}$ band in nadir geometry since a much larger spectral resolution would be required.

As observed with limb spectra in Venus (Gilli et al. 2009) and in limb/nadir sounding in Mars (Formisano et al. 2006; López-Valverde et al. 2005), the nadir dayside IR spectra around $4.3 \mu \mathrm{m}$ also exhibit a characteristic double-peak structure with maxima around 4.28 and $4.32 \mu \mathrm{m}$ (see Fig. 1, and the study of nadir observations with VIRTIS-M by Garcia et al. 2009). These dayside IR spectra are well predicted by NLTE models and are mostly caused by the strong solar pumping at $2.7 \mu \mathrm{m}$ of the $\left(10^{0} 1\right)$ and $\left(02^{0} 1\right)$ vibrational states of the major $\mathrm{CO}_{2}$ isotope (López-Valverde et al. 2007). We use a sophisticated NLTE model of the Venus Atmosphere (Roldán et al. 2000; López-Valverde et al. 2007) to simulate the emerging dayglow emission that would be expected for Venus at different conditions of observations. In the case of nadir 


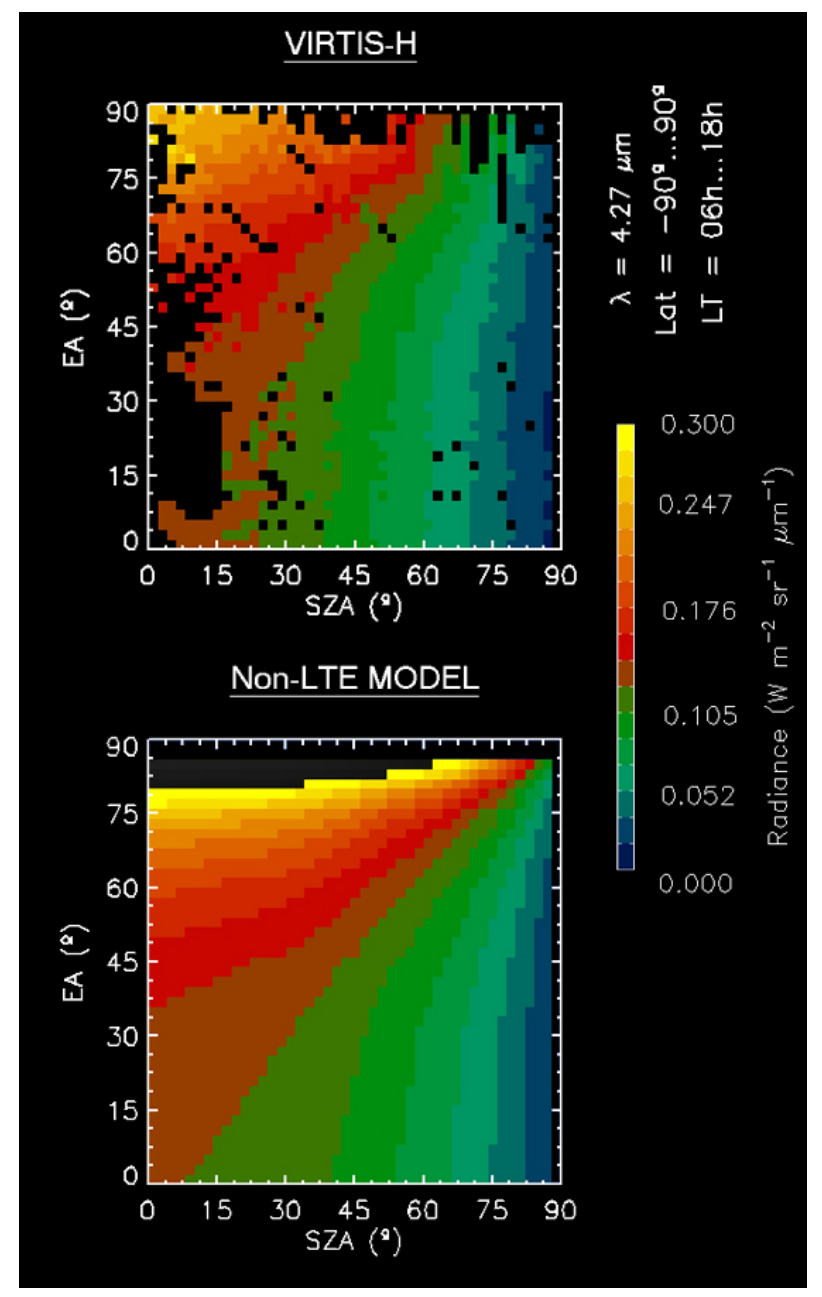

Fig. 2. Comparison between maps SZA-EA of averaged radiance at $4.27 \mu \mathrm{m}$ from spectra taken by VIRTIS-H and from our NLTE model.

observations, it can be demonstrated that the emerging $\mathrm{CO}_{2}$ spectrum at $4.3 \mu \mathrm{m}$ mainly comes from the emitting layers within the range $10^{-2}-10^{-5} \mathrm{mb}$ (see Sect. 3). Figure 1 shows two sets of VIRTIS spectra selected to illustrate the impact of the solar zenith angle (SZA) and of the emission angle (EA). The gradual changes show an enhanced emission for larger solar illumination (lower SZA) and for a larger number of layers contributing to the emission (larger EA). Moreover, the NLTE forward model not only reproduces the spectral shape (Fig. 1), but also the variations with SZA and EA with a good agreement. This is shown in Fig. 2 at $4.27 \mu \mathrm{m}$, a wavelength near the NLTE emission peak. The agreement between model simulations and measurements is satisfactory, including the SZA and emission angle variation (Figs. 1 and 2) except at wavelengths beyond $4.34 \mu \mathrm{m}$. There, a scattering component from the solar reflexion at the clouds' tops, or more likely in the mesospheric hazes, is significant, and the model systematically underestimates the observed radiance. For this reason, the analysis is focused on the range 4.22-4.34 $\mu \mathrm{m}$. This NLTE forward model is a key part of our retrieval scheme. This kind of scheme is similar to that used by Gilli et al. (2015), and consists of two steps described in more detail in Sect. 3.

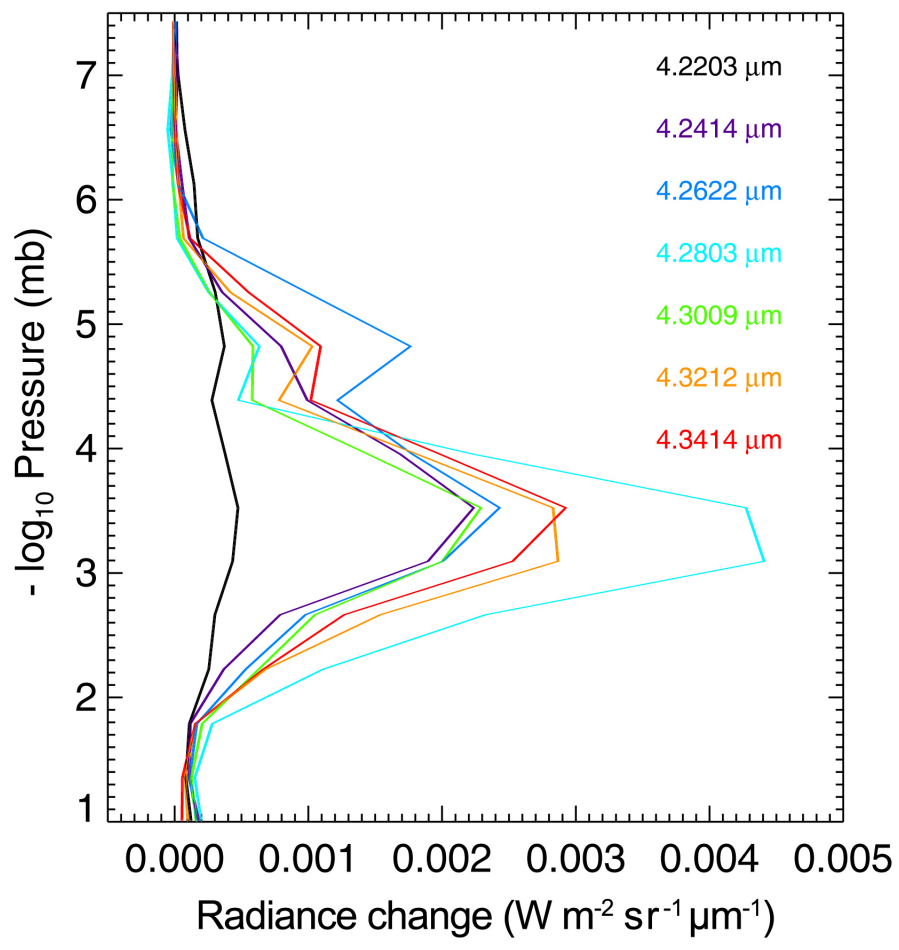

Fig. 3. Sensitivity expected in emerging NLTE emissions at $4.3 \mu \mathrm{m}$ for temperature changes of about $1 \mathrm{~K}$.

\section{Temperature retrieval and error}

As stated in the previous section, we followed a NLTE retrieval scheme previously developed and applied to VIRTIS limb CO emissions by Gilli et al. (2015). In contrast to the work of these authors, where two parameters (temperature and $\mathrm{CO}$ abundance) were derived simultaneously, we only estimated the atmospheric temperature in this case. The core of the inversion scheme is a NLTE forward model that consists of a line-by-line radiative transfer code and the Venus NLTE model developed at IAA/CSIC (Roldán et al. 2000; López-Valverde et al. 2007), which was used to simulate the Venusian emerging infrared dayglow emission for the varied observational conditions of VEx.

Although SZA and EA are the two major parameters defining the emission, the atmospheric temperature may be derived from these VIRTIS-H spectra since the $\mathrm{CO}_{2}$ NLTE nadir emissions still have some sensitivity to temperature (López-Valverde et al. 2005). We tested our NLTE forward model sensitivity for temperature disturbances of about $20 \mathrm{~K}$ at different altitudes, obtaining similar results at most frequencies within the $4.30 \mu \mathrm{m}$ band. These Jacobians, presented Fig. 3, show a peak sensitivity around $5 \times 10^{-4} \mathrm{mb}$, with changes in the radiance of $2-3 \times 10^{-3} \mathrm{~W} \times \mathrm{m}^{-2} \times \mathrm{sr}^{-1} \times \mu \mathrm{m}$, which is about half the nominal noise level for a single spectrum. The width of this function describes the vertical resolution of our retrieval, and hence we cannot resolve narrower features like small-scale waves or thermal gradients within this broad region. Following the previous work by Gilli et al. (2015), we carried out a retrieval of the temperature following two steps.

In the first step, every measured spectrum is compared to a pre-computed set of synthetic spectra, at the appropriate SZA and EA, using a $\chi^{2}$ minimization procedure. This $\chi^{2}$ is evaluated for all the wavelengths in the $4.20-4.35 \mu \mathrm{m}$ range and is used to define a first-fit spectrum. In the second step of the retrieval, a linear inversion is performed around this first fit (used 

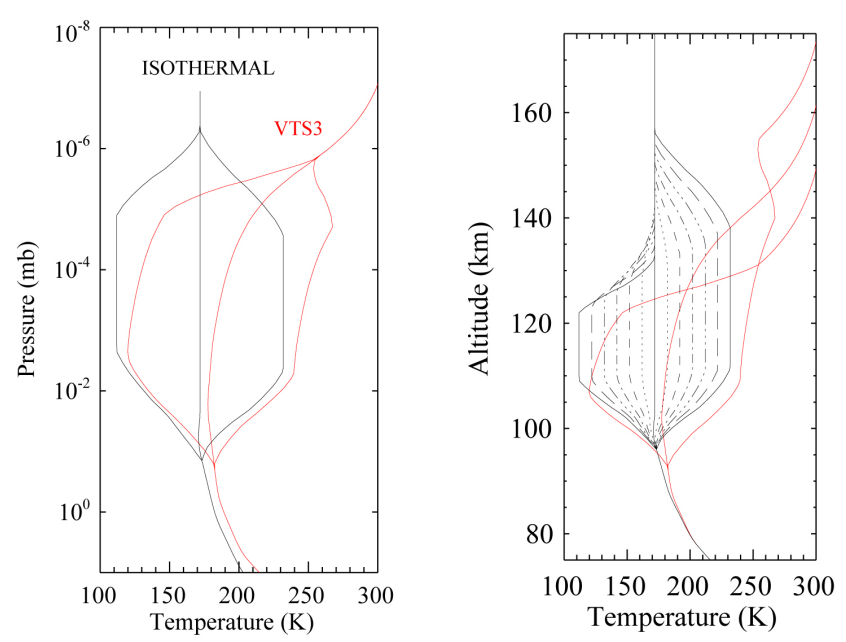

Fig. 4. Thermal profiles used as reference for the set of temperature perturbations in vertical coordinates of pressure $(\mathbf{A})$ and kilometers $(\mathbf{B})$. The red curves corresponds to the thermal profiles from the reference atmosphere VTS3 (Hedin et al. 1983), while the black curve corresponds to an arbitrary isothermal profile. The sensed vertical region for temperature perturbations ranging from \pm 10 to $\pm 60 \mathrm{~K}$ is labelled with different line styles.

as reference state close to the real solution) to obtain the best fit, following the optimal estimation formalism (Rodgers 2000). The set of synthetic spectra was created with the NLTE forward model for a set of atmospheric profiles and observational conditions, with a grid of 11 points in SZA, 11 points in EA, and 13 points in temperature. These 13 temperatures correspond to perturbations from -60 to $+60 \mathrm{~K}$ in regular steps of $10 \mathrm{~K}$ around the nominal VTS3 profile (Hedin et al. 1983) between $10^{-2}$ and $10^{-5} \mathrm{mb}$, as shown in Fig. 4 (left panel). An additional set of 13 thermal profiles was also generated and applied to the $121 \mathrm{ob}-$ servational conditions (11 SZA values times 11 EA values), but with an isothermal reference atmosphere. This isothermal case was used to test the impact of the unknown profile shape on the results, as explained below. Figure 5 shows an example of this two-step retrieval applied to a single VIRTIS-H nadir spectrum.

The whole database of VEx/VIRTIS-H comprised a total of 200, 036 spectra after selecting values of SZA and EA lower than $80^{\circ}$ (the signal-to-noise for higher values of SZA and EA is found to be too low). Despite this restriction, low-quality retrievals were frequently obtained, hence, additional quality criteria were applied. We discarded retrievals where: (a) no clear single minimum was present in the $\chi^{2}$ function; or (b) this minimum was placed at the maximum/minimum temperature perturbation (in our case at $\pm 60 \mathrm{~K}$ ); or (c) the inversion was possibly outside the linear regime, i.e. the absolute value of the difference between the temperatures for best fit and first fit was higher than the step of $10 \mathrm{~K}$ used for temperature perturbations (i.e. $\left.\left|T_{\mathrm{BF}}-T_{\mathrm{FF}}\right|>10 \mathrm{~K}\right)$ or this difference was more than twice the error bars for the best fit. As a result, about a $33 \%$ of the nadir spectra were finally discarded and we obtained 133015 valid retrievals of dayside temperatures covering more than five years of data.

We averaged the atmospheric temperatures hereby obtained for a grid of latitude and local time with bins of $5^{\circ}$ in latitude and $0.25 \mathrm{~h}$ in local time, and chose these values as a compromise between maximizing the spatial coverage and minimizing the errors. The total error in each bin is calculated as
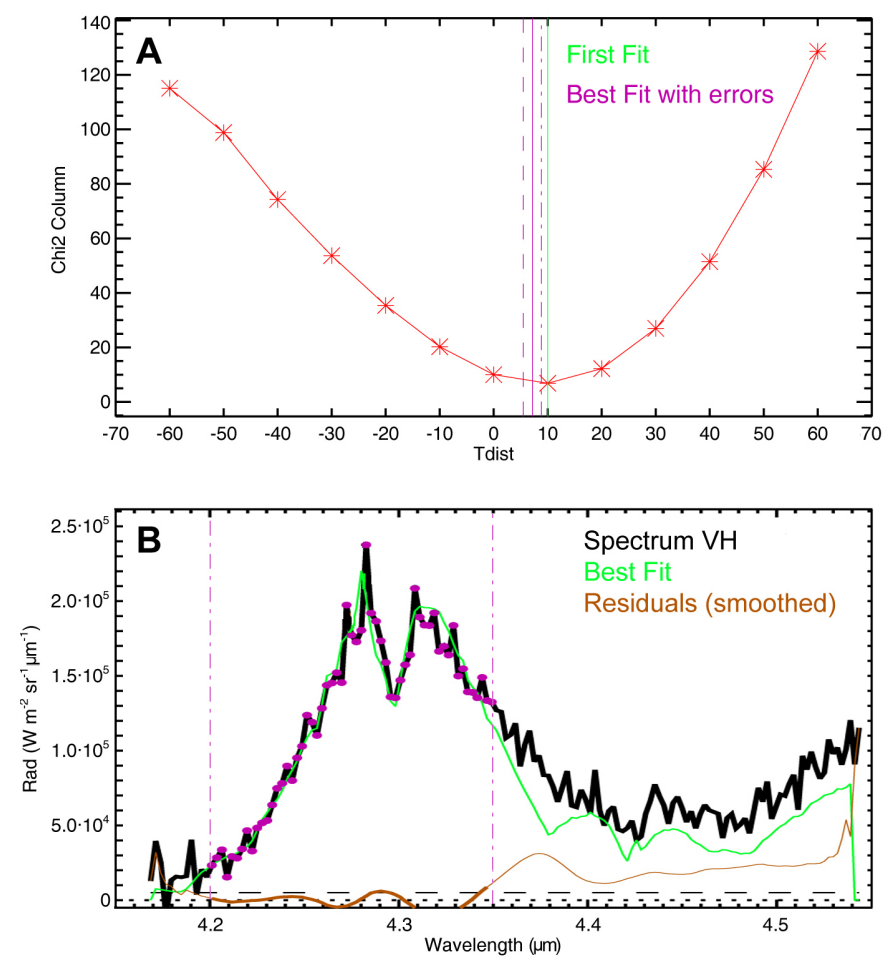

Fig. 5. Model fitting of one VIRTIS-H spectrum. (A) Minimization of $\chi^{2}$ differences for a set of synthetic spectra obtained for 13 different values of atmospheric temperature in the UMLT region, $T_{\text {dist }}$ stands for temperature (K) from a fixed value used as reference; (B) synthetic spectrum best fits an individual nadir spectrum taken by the instrument VIRTIS-H. The fit is carried out using frequencies ranging 4.20$4.35 \mu \mathrm{m}$. The first-step fit and the residuals are also shown with green and brown lines, respectively (see text.)

$\sigma^{2}=\sigma_{\mathrm{SD}}^{2}+\sigma_{\text {Method }}^{2}$, where $\sigma_{\mathrm{SD}}$ is the standard deviation of the temperatures within the bin and the methodological error contains two components: the retrieval error $\left(\sigma_{\text {Ret }}\right)$ and the uncertainty from the unknown shape of the thermal profile used in the set of synthetic spectra $\left(\sigma_{\text {Shape }}\right)$. As mentioned above, the actual shape of the thermal profile is unknown within the range of altitudes (pressures, to be precise) actually sounded by this $\mathrm{CO}_{2}$ $4.3 \mu \mathrm{m}$ band. To evaluate the impact that this uncertainty has on the temperature retrieval, two different profiles were used when generating the database of pre-computed spectra. One of these is the VTS3 daytime profile (Hedin et al. 1983), possibly more appropriate for near-subsolar soundings, and the second one is a colder and isothermal profile, presumably closer to higher SZA and near-terminator conditions. Both profiles were disturbed in a similar way, with $10 \mathrm{~K}$ steps within the pressure range of interest, $10^{-2}-10^{-5} \mathrm{mb}$. Figure 4 shows these reference atmospheres in isobaric coordinates (left-hand panel). Nevertheless, a word of caution is needed for comparisons with other results. Our retrieval scheme is well defined in a pressure scale but the actual altitudes may change depending on the actual thermal structure. This is clearly illustrated in the right-hand panel of Fig. 4, which is similar to the left-hand panel except in altitude coordinates. This profile-shape uncertainty $\left(\sigma_{\text {Shape }}\right)$ turned out to be larger than the retrieval error $\left(\sigma_{\text {Ret }}\right)$, especially near the terminator (high latitudes, and early morning and late afternoon local times). In addition, the joint contribution or methodological error 

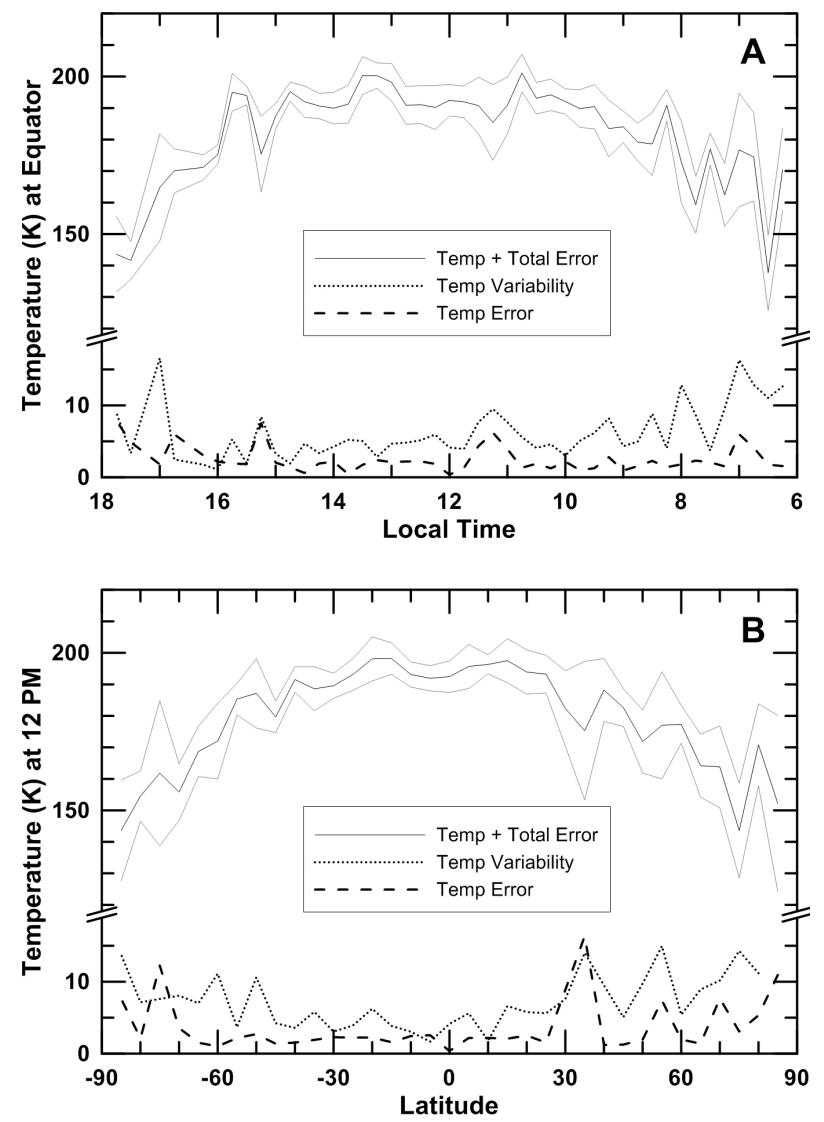

Fig. 6. Comparison for typical errors at the equator A) and at midday B). The averaged temperature along with the total error is shown with continuous lines, while the error due to the variability (standard deviation of the mean) and that due to the pure error (retrieval error plus the nominal profile dependent) are shown with dotted and dashed lines, respectively.

$\left(\sigma_{\text {Method }}^{2}\right)$ is usually smaller than the actual atmospheric variability $\left(\sigma_{\mathrm{SD}}^{2}\right)$. All these error terms are shown in Fig. 6.

\section{Results and discussion}

After applying specific criteria to discard lower quality retrievals (see Sect. 3), we obtained a total of 133015 dayside temperatures covering more than five years of data (from 2006/05/14 to 2011/06/05). These errors are typically between 5-10 K with a slight increase towards the terminator (see Sect. 3). Also, as explained previously, these temperatures were averaged onto a grid of latitude and local time with bins of $5^{\circ}$ in latitude and $0.25 \mathrm{~h}$ in local time to maximize the spatial coverage and minimize errors. The number of spectra or temperatures in most of these boxes varies between 5 and 20, with a few boxes with more than 25 values. The magnitude of the standard deviation within each bin is about $15 \mathrm{~K}$, interpreted to be caused by the atmospheric variability. This is similar to typical noise values. The exception is close to the terminator, where the standard deviation has a small increase, up to 20-25 K. Figure 7A exhibits the obtained 2D horizontal distribution of temperature and Fig. 8 shows the latitude and local time variations (meridional and zonal scans in the 2D map) at the equator. A strong gradient of temperature of about $55 \mathrm{~K}$ is apparent from the warmest area at the subsolar point (around 190-200 K) and decreases to about $140 \mathrm{~K}$ at the near terminator.
This result is in qualitative agreement with the Venus' upper atmosphere global models. Figure 7 also shows latitudelocal time maps of numerical results from the Venus Thermal General Circulation Model (VTGCM) by Brecht \& Bougher (2012) (panel B) and its difference from our measurements (C), while Fig. 8 shows the variations of this model with latitude (panel A) and with local time (B) around the subsolar point. Their simulations were performed for solar cycle conditions representative of the VEx data set (near solar minimum). For a coherent comparison, data from the VTGCM were averaged for the interval $10^{-2}-10^{-5} \mathrm{mb}$ with our Jacobian functions (see Fig. 3) as well as interpolated onto the same grid of latitude and local time as VIRTIS-H. Figures 7 and 8 clearly exhibit a colder atmosphere than in the VTGCM, with temperatures reaching differences of 20-25 K near the subsolar point that were smaller away from this point and increasing up to $30 \mathrm{~K}$ at the near terminator, although our data and retrievals here are noisier. The standard deviation in the VTGCM bins is maximum at the subsolar point, about $25 \mathrm{~K}$, and smaller, about $15 \mathrm{~K}$, near the terminator. Our data presents a plateau around the subsolar point, in contrast to the model that shows a clear maximum in the central point. This difference appears to be above the noise level and it might be the result of atmospheric variability, mostly temporal variability in our data set. This difference has been also observed in limb observations of the $\mathrm{CO}$ dayglow with retrieved temperatures lower than the VTGCM in the subsolar region (Gilli et al. 2015, see Fig. 14). Nightside temperatures from SPICAV (Piccialli et al. 2015) were also averaged for the same pressure interval as our dayside data (see panel 7D), exhibiting a much colder atmosphere.

A comparison with other temperature measurements is shown in Figs. 7 and 8, and a study of the time evolution in our data is also indicated in Fig. 8. Concerning the temperatures derived from the $\mathrm{CO}$ dayglow measured by VIRTIS-H in the limb (Gilli et al. 2015) a good agreement is found despite their large error bars (above $40 \mathrm{~K}$ ). Regarding temperatures from groundbased observations (Krasnopolsky 2014) a proper comparison is not possible because of the different vertical layer sensed. And although solar occultation with SOIR show larger error bars (Mahieux et al. 2015a), and their vertical weighting functions are also different, their temperatures agree well with the tendency of our values towards the terminator. On the other hand, the thermal gradient between the subsolar and antisolar meridians is of crucial importance to get an accurate evaluation of the SS-AS circulation. Caution must be taken in comparing VIRTIS with SPICAV data, since SPICAV uses altitude as the vertical coordinate. For this reason, we used Fig. 4 to infer which is the corresponding altitude interval sensed for the subsolar temperatures of about $190 \mathrm{~K}$ (in this case, 100-125 km). Accordingly, SPICAV temperatures for the antisolar region were averaged for the same altitude region. Subsolar and antisolar values (combining VIRTIS and SPICAV data) are presented in Fig. 8C and show a difference of $33 \pm 21 \mathrm{~K}$, much lower than the VTGCM large day-night differences of more than $50 \mathrm{~K}$ at a fixed altitude like $110 \mathrm{~km}$ (Brecht \& Bougher 2012). However, the temperature in the nightside varies a lot with altitude within our pressure range and careful model averages should be considered. For example, the model differences between the mesopeak on the dayside (around $110 \mathrm{~km}$ ) and on the nightside (around $104 \mathrm{~km}$ ) is about $40 \mathrm{~K}$, closer to our day-night gradient.

Finally, we examined long-term and short-term variations in the UMLT region. The time variation of the mean temperature for latitudes $30^{\circ} \mathrm{S}-30^{\circ} \mathrm{N}$ of the subsolar region $(10 \mathrm{~h}-14 \mathrm{~h})$ and evening (14h-16h) is shown along years (Fig. 8D) and days 

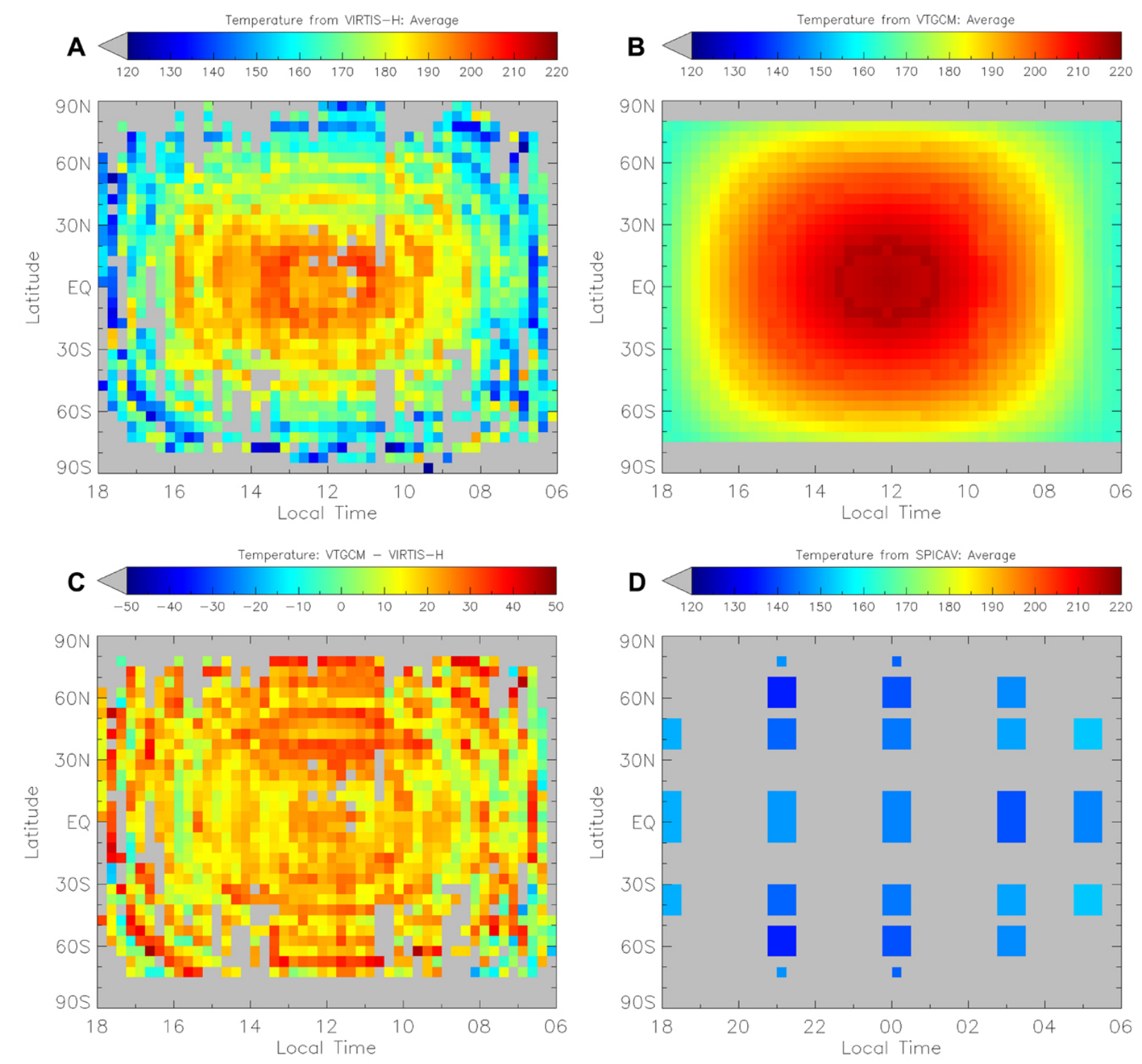

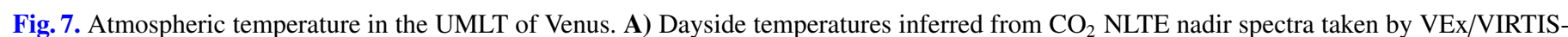
$\mathrm{H}$; B) dayside temperatures from the VTGCM by Brecht \& Bougher (2012) and averaged for the pressure interval $10^{-2}-10^{-5} \mathrm{mb}$; C) difference between temperatures from the VTGCM and VIRTIS-H; D) nightside temperatures inferred with stellar radio-occultation data from VEx/SPICAV (Piccialli et al. 2015) and averaged for the pressure interval $10^{-2}-10^{-5} \mathrm{mb}$.

(Figs. 8E). In the case of the long-term behaviour, temperatures have been also averaged along series of consecutive days covering no more than 40 days, depending on the availability of data. For the short-term behaviour, we only display daily averages. The basic result is that VIRTIS data exhibit a fairly stable behaviour, at least on the dayside hemisphere and on our averaged UMLT region. In contrast to the apparent stability exhibited for temperatures at midday and evening zones, remarkable variations up to $30 \mathrm{~K}$ seem trigger in only one or two days in the subsolar region as shown with blue areas in panel E. These sudden thermal variations are also compared in panels $\mathrm{D}$ and $\mathrm{E}$ with the solar radio flux at $10.7 \mathrm{~cm}$ (Tobiska et al. 2000), with no apparent correlation.

\section{Conclusions}

In this paper, we have measured for the first time the horizontal distribution of the dayside temperatures in the UMLT of Venus at the pressure interval $10^{-2}-10^{-5} \mathrm{mb}$. A total of 133015 dayside temperatures covering more than five years of data have been obtained by means of an inversion procedure applied to the NLTE $\mathrm{CO}_{2}$ dayglow nadir spectra at $4.3 \mu \mathrm{m}$, as measured by the instrument VIRTIS-H on board VEx. Our dayside temperatures peak is about $195 \mathrm{~K}$ in a broad region around the subsolar point, extending to the latitude interval $20^{\circ} \mathrm{S}-20^{\circ} \mathrm{N}$ and to local times $10 \mathrm{~h}-15 \mathrm{~h}$. This is in contrast to VTGCM results, which peak precisely at the subsolar point and reach $215 \mathrm{~K}$. When there is coincidence, our results are in good agreement with the few previous ground-based and remote sensing measurements available at these altitudes. Our results behave similarly to predictions from numerical models (Brecht \& Bougher 2012), and decrease away from the subsolar point, although they exhibit a $25 \mathrm{~K}$ colder atmosphere. A gradient of up to $35 \mathrm{~K}$ is found between the subsolar and antisolar points when comparing our data with nightside temperatures from SPICAV at the same altitude region (Piccialli et al. 2015). Finally, time evolution shows that UMLT temperatures on Venus are very stable through the years 

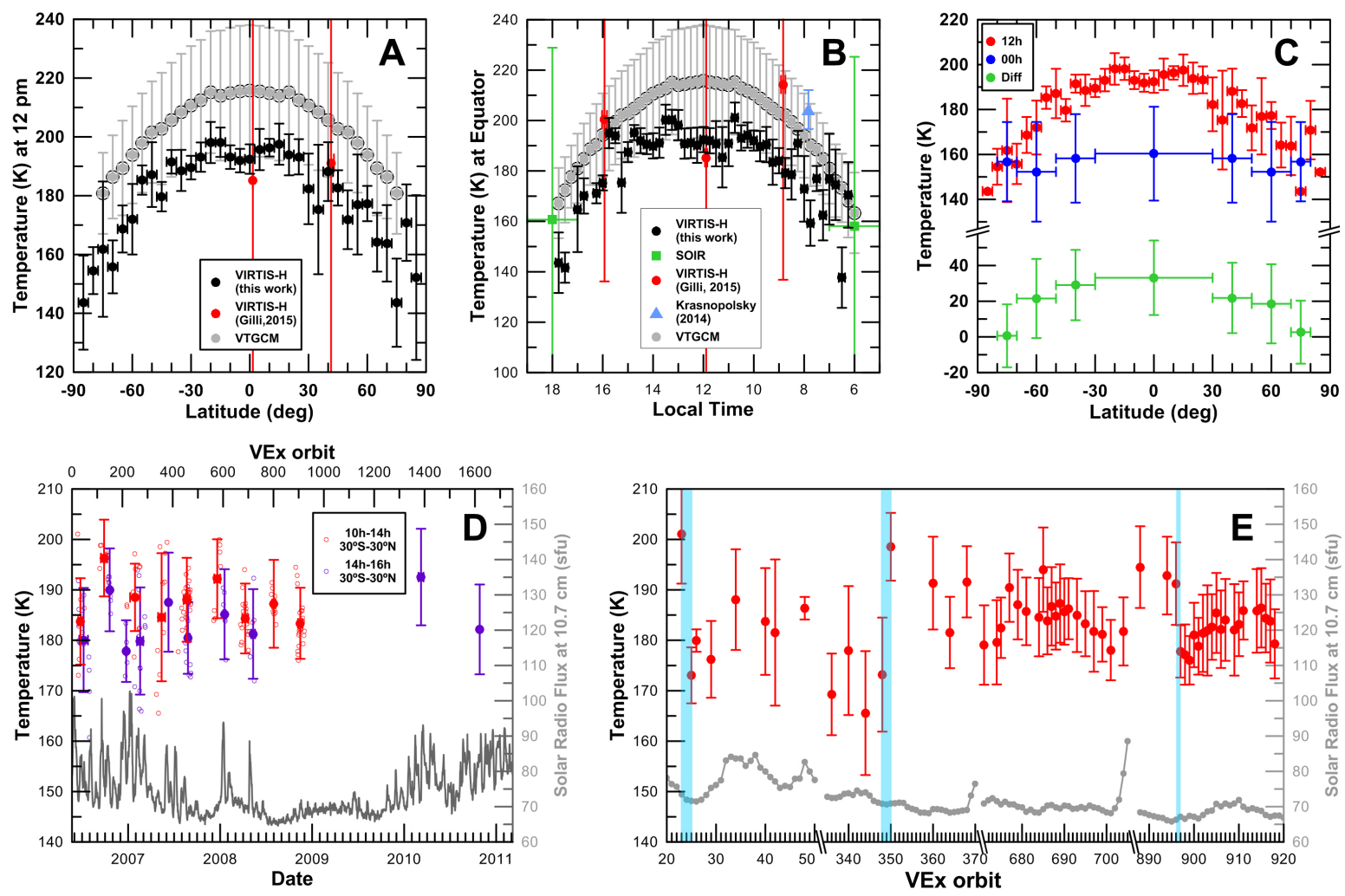

Fig. 8. Atmospheric temperature at the lower thermosphere of Venus: comparison and time evolution. A) and B) show our temperatures at the subsolar meridian and the equator compared with results from CO NLTE limb spectra (Gilli et al. 2015; Krasnopolsky 2014), solar occultation with SOIR/VEx (Mahieux et al. 2015a), and numerical VTGCM (Brecht \& Bougher 2012); C) subsolar (red) and antisolar temperatures (blue) inferred with $\mathrm{CO}_{2}$ NLTE spectra taken by VEx/VIRTIS-H (this work) and with stellar occultation using SPICAV (Piccialli et al. 2015). The difference between subsolar and antisolar temperatures are shown in green. Antisolar temperatures are averaged for the same altitude region as the pressure layer $10^{-2}-10^{-5} \mathrm{mb}$ at the subsolar meridian (see Fig. 4); D) and E) indicate long- and short-time evolution of the temperature, averaged for consecutive and single days, respectively, as well as for intervals of latitude $\left(30^{\circ} \mathrm{S}-30^{\circ} \mathrm{N}\right)$ and local time $(10 \mathrm{~h}-14 \mathrm{~h}$ shown with red dots, and $14 \mathrm{~h}-16 \mathrm{~h}$ with purple dots). The solar radio flux at $10.7 \mathrm{~cm}$ (Tobiska et al. 2000) is also shown in grey. Sudden changes in temperature are shown in cyan in panel $\mathbf{E})$.

except for several episodes where the subsolar region is shown to vary about $30 \mathrm{~K}$ in less than two days by mechanisms yet to be unveiled.

Acknowledgements. J.P. and MA.L-V acknowledge the Spanish MICINN for funding support through the CONSOLIDER program "ASTROMOL" CSD200900038 and project AYA2011-23552. J.P. also thanks the JAXA International Top Young Fellowship program, GG thanks CNES postdoc contract and A.P. acknowledges funding from the European Union Seventh Framework Programme (FP7/2007-2013) under Grant agreement No. 246556. We also thank J. L. Bertaux and F. Montmessin for their support with VEx/SPICAV.

\section{References}

Bougher, S. W., Roble, R. G., \& Fuller-Rowell, T. 2002, Washington DC American Geophysical Union Geophysical Monograph Series, 130, 261 Bougher, S. W., Rafkin, S., \& Drossart, P. 2006, Planet. Space Sci., 54, 1371 Brecht, A. S., \& Bougher, S. W. 2012, J. Geophys. Res. (Planets), 117, 8002 Dickinson, R. E. 1972, J. Atmos. Sci., 29, 1531

Drossart, P., Piccioni, G., Adriani, A., et al. 2007, Planet. Space Sci., 55, 1653 Formisano, V., Maturilli, A., Giuranna, M., D’Aversa, E., \& Lopez-Valverde, M. A. 2006, Icarus, 182, 51

Garate-Lopez, I., García Muñoz, A., Hueso, R., \& Sánchez-Lavega, A. 2015, Icarus, 245, 16

Garcia, R. F., Drossart, P., Piccioni, G., López-Valverde, M., \& Occhipinti, G. 2009, J. Geophys. Res. (Planets), 114, 0

Gilli, G., López-Valverde, M. A., Drossart, P., et al. 2009, J. Geophys. Res. (Planets), 114, E00B29

Gilli, G., López-Valverde, M. A., Peralta, J., et al. 2015, Icarus, 248, 478

Grassi, D., Migliorini, A., Montabone, L., et al. 2010, J. Geophys. Res., 115, 9007
Hedin, A. E., Niemann, H. B., Kasprzak, W. T., \& Seiff, A. 1983, J. Geophys. Res., 88, 73

Keating, G. M., Bertaux, J. L., Bougher, S. W., et al. 1985, Adv. Space Res., 5, 117

Krasnopolsky, V. A. 2014, Icarus, 237, 340

López-Puertas, M., \& Taylor, F. W. 2001, in Non-LTE radiative transfer in the atmosphere (World Scientific), eds. M. López-Puertas, \& F. W. Taylor, 3

López-Valverde, M. A., López-Puertas, M., López-Moreno, J. J., et al. 2005, Planet. Space Sci., 53, 1079

López-Valverde, M. A., Drossart, P., Carlson, R., Mehlman, R., \& Roos-Serote, M. 2007, Planet. Space Sci., 55, 1757

Mahieux, A., Vandaele, A. C., Bougher, S. W., et al. 2015a, Planet. Space Sci., 113,309

Mahieux, A., Vandaele, A. C., Robert, S., et al. 2015b, Planet. Space Sci., $113-$ 114,347

Migliorini, A., Grassi, D., Montabone, L., et al. 2012, Icarus, 217, 640

Piccialli, A., Montmessin, F., Belyaev, D., et al. 2015, Planet. Space Sci., 113, 321

Rodgers, C. D. 2000, Inverse Methods for Atmospheric Sounding - Theory and Practice. Series: Series on Atmospheric Oceanic and Planetary Physics (World Scientific Publishing Co. Pte. Ltd.), 2, 2

Roldán, C., López-Valverde, M. A., López-Puertas, M., \& Edwards, D. P. 2000, Icarus, 147,11

Sonnabend, G., Krötz, P., Schmülling, F., et al. 2012, Icarus, 217, 856

Svedhem, H., Titov, D. V., McCoy, D., et al. 2007, Planet. Space Sci., 55, 1636

Tellmann, S., Pätzold, M., Häusler, B., Bird, M. K., \& Tyler, G. L. 2009, J. Geophys. Res. (Planets), 114, E00B36

Tellmann, S., Häusler, B., Hinson, D. P., et al. 2012, Icarus, 221, 471

Tobiska, W. K., Woods, T., Eparvier, F., et al. 2000, J. Atmos. Solar-Terr. Phys., 62,1233

Wilquet, V., Fedorova, A., Montmessin, F., et al. 2009, J. Geophys. Res., 114, E00B42 